\title{
Impacted Intra Sphenoid Foreign Body in an Adult: A Rare Event and a Lucky Survivor
}

SOURABHA KUMAR PATRO, ${ }^{1}$ ROSHAN VERMA,${ }^{1}$ NARESH K PANDA ${ }^{1}$

\begin{abstract}
Nasal foreign bodies are rare events occurring mostly in children. However they can be seen in adults as a result of trauma resulting from road traffic accidents and bullet injuries. However these foreign bodies, if associated with high velocity, can cause greater harm at skull base by casing injury to vital structures. Hence safe planning and removal is warranted. We present a case of a 48 year old female with an impacted metallic foreign body in the sphenoid. Endoscopic removal of the foreign body was was done.
\end{abstract}

Key words: sphenoid sinus foreign body, metallic foreign body, paranasal sinus foreign body, endoscopic removal of foreign bodies.

\section{Introduction}

Nasal foreign bodies are an uncommon occurrence and are a more common event in children compared to adults. ${ }^{1}$ Unlike foreign bodies at other sites of body nasal foreign bodies can go unnoticed due to lack of symptoms. ${ }^{2}$ Foreign bodies in nose is usually because of curiosity and a whim to explore orifices mostly seen in children, playful insertion of foreign bodies into others' body parts, accidental entry of foreign body, preexisting disease causing irritation and habitual cleaning of ear and nose with object like ear buds. ${ }^{3,4}$ Of all the sinuses, foreign bodies of frontal and maxilla are common than those found in ethmoids and sphenoid sinuses. ${ }^{5,6}$ Path of any foreign body to reach the sphenoid sinus can be through the orbit or the nasal cavity. ${ }^{7}$ Close proximity of vital structures like optic nerve, internal carotid artery and sella make sphenoid injury and sphenoidal foreign bodies potentially life threatening. ${ }^{5,8}$ Endoscopic endo-nasal approach is usually used for such foreign bodies and considered to be a better approach for this purpose because of the magnification and illumination is achieved with the endoscopes place and added advantage for the same. ${ }^{9-11}$ However rarely an open approach may be needed for such foreign bodies. ${ }^{10}$ Use of endoscopic approach requires preoperative planning and sound knowledge of the anatomy of the sphenoid sinus in view of the presence of the various vital structures in the region. ${ }^{12}$ We present a case of neglected foreign body in sphenoid sinus in an adult female who presented to us after an alleged history of an accidental fall.

1. Department of Otolaryngology, Head and Neck surgery, Post Graduate Institute of Medical Education and Research, Chandigarh, India- 160012.

Correspondence: Dr. Roshan K Verma, Assistant Professor, Department of Otolaryngology, Head and Neck surgery, Post Graduate Institute of Medical Education and Research, Chandigarh, India-160012.Phone -91-9914209761, Fax - +91-172-2744401, E-mail: roshanverma@hotmail.com

\section{Case Report}

A 48 year old female patient was referred to us from the department of ophthalmology for evaluation and management after repair of an open globe defect. The patient presented with an alleged history of fall in the back yard in on a metallic object as a result of a house hold accident after being slipped on a wet floor 10 days back. Patient sustained an open globe injury and was treated for the same in the department of ophthalmology. Primary survey of the patient had shown no other injury anywhere else in the body.

Routine head to toe examination and basic investigations like hemogram, coagulogram, serum electrolytes, chest X ray, ECG, USG abdomen done in FAST(focused assessment with sonography in trauma) and Computerized tomography (CT) scan of head showed no significant findings. However CT paranasal sinuses and orbit showed a hyper dense area in the skull base in the reason of the floor of the sphenoid sinus with hetero-dense soft tissue density in the sphenoid sinus (Figure 1). The density of the lesion was found to be equal to that of the bone.
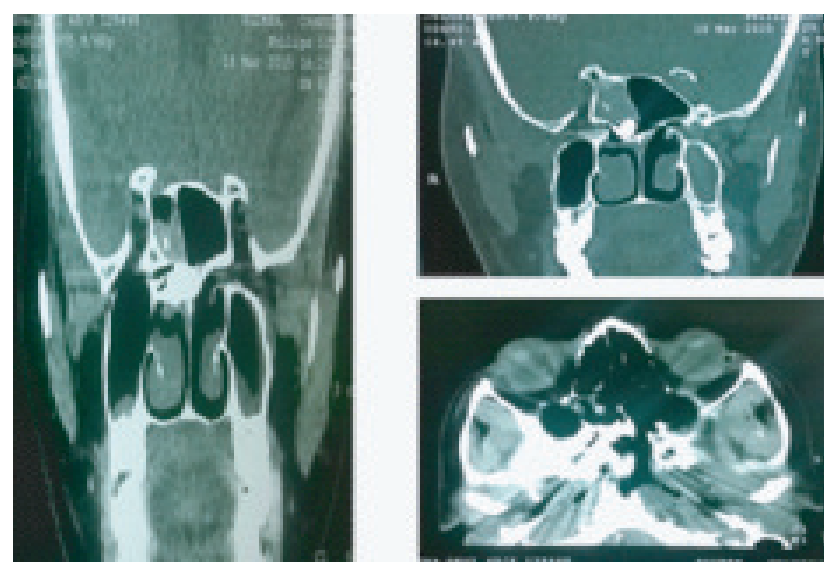

Fig.-1: Computerized tomographic pictures of the foreign body seen in the floor of the sphenoid sinus. 


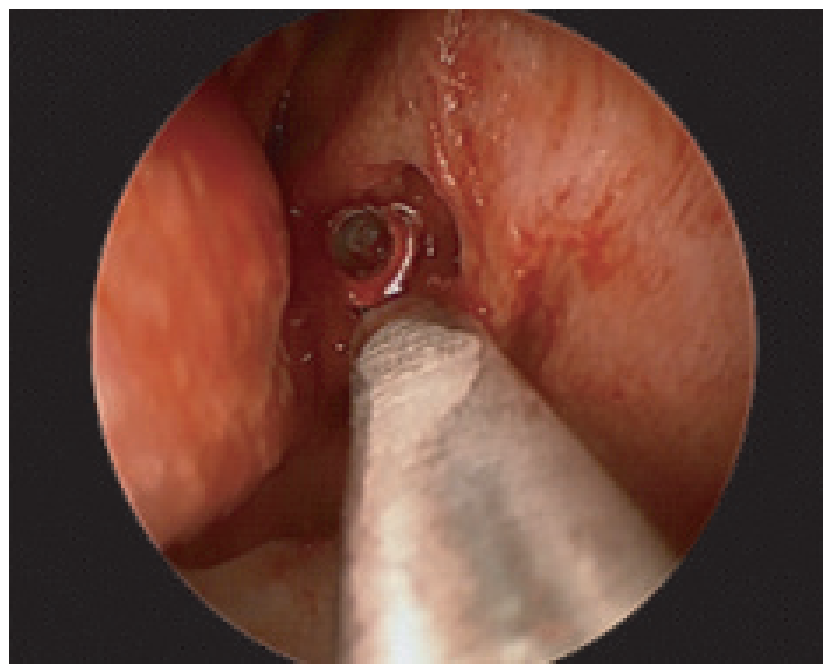

Fig.-2: Impacted foreign body in the floor of the sphenoid sinus and the natural ostium of the sphenoid sinus.

A provisional diagnosis of sphenoid sinus hematoma with suspected foreign body in the floor of the sphenoid sinus was made. Diagnostic nasal endoscopy did not reveal any evidence of trauma in the nasal cavity with intact mucosa in the nasal cavity. There was a presence of concha bullosa both in the endoscopy and the CT scan on the suspected side which obscured the further view during the diagnostic nasal endoscopy (Figure 2). The patient was planned and taken for endoscopic surgery under general anesthesia for the same.

Under general anesthesia with orotracheal intubation and ventilation, $0^{\circ}, 40^{\circ}$ and $90^{\circ}$ endoscopes were used for the surgery.Adequate decongestion and lateral laminectomy for the concha bullosa was performed to improve the access to the spheno-ethmoidal recess and recess was approached medial to the middle turbinate. After suctioning of the secretions in the spheno-ethmoidal recess, a metallic foreign body could be identified impacted in the floor of the sphenoid sinus. Natural ostium of the sphenoid was identifiedand widened. The foreign body was delineated and disimpacted and gently manipulated to bring it outside the sphenoid ostium which was removed with forceps.(figure 3) Post removal sphenoid ostium was widened and intrasphenoid hematoma was sucked and hemostasis achieved.

\section{Discussion}

Foreign bodies are rare entities in paranasal sinuses. It is more common in frontal and maxilla compared to sphenoid and ethmoid sinuses. Sphenoid sinus is rarely involved and most of the times it is a metallic one associated with orbital and maxillofacial trauma. ${ }^{1,4,12}$ In this case our patient was

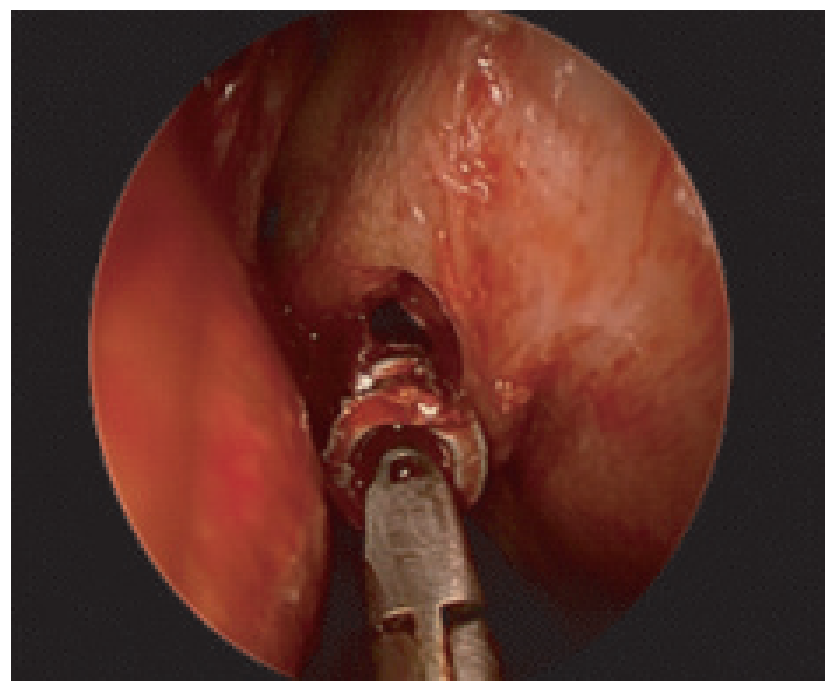

Fig.-3: Dis impaction of the foreign body done with a periosteum elevator and its removal with the help of tilley's forceps.

also exposed to fall leading to accidental high velocity insertion of the foreign body in to sphenoid sinus. Route of entry is usually though the orbit or the nasal cavity for the sphenoid sinus foreign bodies. ${ }^{7}$ In this case it was suspected to have followed a trans nasal route as though there was an associated open globe injury, but there was no evidence of any definite tract showing trans orbital entry of the foreign body.

Radiological investigations play a crucial role in the diagnosis of the foreign bodies in the paranasal sinuses. CT scan is an useful tool for the diagnosis of sphenoid sinus foreign bodies and to know its relation with the surrounding structures and any evidence of injury to surrounding orbit, internal carotid artery(ICA), optic nerve and sella. ${ }^{7}$ In the present case we also resorted to computerized tomographic scans for the diagnosis of the foreign body and to access the nature of injury to the surrounding structures. However literature does provide evidence for the use of magnetic resonance imaging for diagnosis of nonmetallic foreign bodies, where CT scans are less informative. ${ }^{89}$ Digital subtraction angiography (DSA) has also been used by an author in the literature to rule out any vascular injury before attempting removal of an sphenoid sinus foreign body. ${ }^{13}$

Intracranial penetrations of foreign bodies at the primary setting or secondarily by migration can cause sudden intracranial complications like subarachnoidal or intraparenchymal cerebral hemorrhages, CSF rhinorrhea and pneumocephalus as well as delayed severe complications including meningitis or cerebral abscess and any such complication warrants an immediate removal of the foreign body by an endoscopic or open approach. ${ }^{12}$ Risk of vascular 
event such as formation of pseudo aneurysms or rupture of ICA can also occur by sphenoid sinus foreign bodies leading to catastrophic events. All foreign bodies act as source and nidus of infection at the site of lodgment. Because of the close relationship between the sphenoidal sinus and the optic nerve, ${ }^{14}$ cavernous sinus, ICA and other important structures of the skull base, sphenoidal sinusitis secondary to a foreign body may cause catastrophic results. ${ }^{13}$ This patient was lucky by not having any such complications.

Best surgical approach to sphenoid sinus remains endoscopic due to the increased illumination and magnification that can be achieved by the modern day endoscopy. $8,11,15$ However rarely open approaches are also needed for the purpose of removal of foreign bodies in the spheno-ethmoidal region. ${ }^{10,16}$ In the present case the foreign body was found to be located in floor of the sphenoid sinus in the CT scan and hence an endoscopic approach was used for the removal of the foreign body and it could be safely removed without any procedure related complication or sequel. Endoscopic technique has also the advantage of feasibility of repair of the skull base defects, if caused by the foreign body. ${ }^{13}$

\section{Conclusion}

Sphenoid sinus foreign bodies are rare incidences that an otolaryngologist meets with during his clinical endeavor. They can be detected late due to the absence of symptoms as compared to the foreign bodies of other sites of the body. They can be hazardous at the primary setting or secondarily by dislodgement or infections due to the presence of various vital structures near the sphenoid sinus, hence warrants removal. With the recent development of improved instrumentation and visualization in the modern day endoscopic techniques, endoscopic endo-nasal approach proves as an effective and safe technique for removal of these foreign bodies and management of any skull base defects produced by the foreign body.

\section{Conflict of interest: None}

\section{References:}

1. Alsarraf R, Bailet JW. Self-inserted sphenoid sinus foreign bodies. Archives of otolaryngology — head \& neck surgery 1998;124(9):1018-20.

2. Kelesidis T, Osman S, Dinerman H. An unusual foreign body as cause of chronic sinusitis: a case report. Journal of medical case reports 2010;4:157.

3. Das SK. Aetiological evaluation of foreign bodies in the ear and nose. The Journal of laryngology and otology 1984;98(10):989-91.
4. SrinivasMoorthyPN, Srivalli M, Rau GV, Prasanth C. Study on clinical presentation of ear and nose foreign bodies. Indian journal of otolaryngology and head and neck surgery. 2012;64(1):31-5.

5. Dimitriou C, Karavelis A, Triaridis K, Antoniadis C. Foreign body in the sphenoid sinus. Journal of cranio-maxillo-facial surgery. 1992;20(5):228-9.

6. O'Connell JE, Turner NO, Pahor AL. Air gun pellets in the sinuses. The Journal of laryngology and otology 1995;109(11):1097-100.

7. Wani NA, Khan AQ. Foreign body within sphenoid sinus: multidetector-row computed tomography (MDCT) demonstration. Turkish neurosurgery 2010;20(4):547-9.

8. Kitajiri S, Tabuchi K, Hiraumi H. Transnasal bamboo foreign body lodged in the sphenoid sinus. Auris, nasus, larynx 2001;28(4):365-7.

9. LaFrentz JR, MairEA, Casler JD. Craniofacial ballpoint pen injury: endoscopic management. The Annals of otology, rhinology, and laryngology 2000;109(2):119-22.

10. Kayikcioglu A, Karamusel S, Mavili E, Erk Y, Benli K. Intrasphenoidal migration of a premaxillaryKirschner wire. The Cleft palate-craniofacial journal. 2000;37(2):209-11.

11. Bhattacharyya N, WenokurRK. Endoscopic management of a chronic ethmoid and sphenoid sinus foreign body. Otolaryngology—-head and neck surgery. 1998;118(5): 687-90.

12. Presutti L, Marchioni D, Trani M, Ghidini A. Endoscopic removal of ethmoido-sphenoidal foreign body with intracranial extension. Minimally invasive neurosurgery: MIN 2006;49(4):244-6.

13. Yildirim AE, Divanlioglu D, Cetinalp NE, Ekici I, Dalgic A, Belen AD. Endoscopic endonasal removal of a sphenoidal sinus foreign body extending into the intracranial space.Ulusaltravmaveacilcerrahidergisi $=$ Turkish journal of trauma \& emergency surgery :TJTES 2014;20(2):139-42.

14. DeLano MC, Fun FY, ZinreichSJ. Relationship of the optic nerve to the posterior paranasal sinuses: a CT anatomic study. AJNR. American journal of neuroradiology. 1996;17(4): 669-75.

15. Liu Z, Lin Y, Zhang L, Yang C, ShenSG, Wang X. Endoscopic-Assisted Navigation-Guided Removal of Longstanding Metallic Foreign Body Near to the Sphenoid. The Journal of craniofacial surgery. 2015;26(2):e122-4.

16. Sharma R, Minhas R, Mohindroo N. An unusual foreign body in the paranasal sinuses.Indian journal of otolaryngology and head and neck surgery. 2008;60(1): 88-90. 